\title{
Téoros
}

Revue de recherche en tourisme

\section{Essai bibliographique et pistes de recherche}

\section{Daniel Dumas}

Volume 4, numéro 1, mars 1985

\section{L'information touristique}

URI : https://id.erudit.org/iderudit/1080745ar

DOI : https://doi.org/10.7202/1080745ar

Aller au sommaire du numéro

\section{Éditeur(s)}

Université du Québec à Montréal

\section{ISSN}

0712-8657 (imprimé)

1923-2705 (numérique)

Découvrir la revue

\section{Citer ce document}

Dumas, D. (1985). Essai bibliographique et pistes de recherche. Téoros, 4(1), 26-27. https://doi.org/10.7202/1080745ar d'utilisation que vous pouvez consulter en ligne.

https://apropos.erudit.org/fr/usagers/politique-dutilisation/ 


\section{Essai bibliographique et pistes de recherche}

par Daniel Dumas

L'avantage d'un essai bibliographique est qu'il permet de mettre en évidence les lacunes et les possibilités de la recherche. Le domaine de l'information touristique, comme on le constatera dans la mince bibliographie qui suit, est encore peu étudié. Pourtant l'information touristique soulève des questions et offre des prises intéressantes pour la recherche. Quel que soit le modẻle théorique ou l'approche adoptés, une analyse du contenu des cahiers touristiques des quotidiens ou des chroniques de voyages des magazines pourrait être intéressante à plus d'un titre. Pourquoi pas une analyse du message avec ses stéréotypes, ses biais culturels comme aussi bien ses comportements d'ouverture? Une analyse des politiques éditoriales de la répartition des contenus, révélateurs d'in- térêts économiques, culturels ou politiques? Ou encore une étude de la pratique journalistique avec ses contraintes (bien particulières dans le domaine du tourisme), son éthique et son debat entre subjectivité et objectivité qui se pose de façon originale en tourisme? Enfin l'information touristique peut être étudiée du bas vers le haut, c'està-dire du point de vue du récepteur, de ses besoins et de ses droits. Ce ne sont là que quelques pistes relevées au fil du constat bibliographique qui suit.

Cette courte bibliographie se présente en deux volets. Le premier recense les études et lés textes de réflexion sur le phénomène de l'information touristique. Le relatif vide documentaire sur le sujet noụs a obligé à $y$ inclure autant les courts textes d'une page que les quelques monographies bien documentées.

Le second volet est un rapide inventaire de l'information touristique. II s'agit du corpus sur lequel pourrait se pencher d'éventuels chercheurs. Le manque d'espace nous a ici obligé â ne pas signaler certains phénomènes (par exemple: les chroniques de voyages des magazines). Un inventaire exhaustif de l'information touristique (sous toutes ses formes) reste à faire.

Tous les documents de cette bibliographie sont disponibles pour consultation au Centre d'études du tourisme, sauf ceux marqués d'un * qui dont disponibles au Centre de recherches et de données touristiques de Tourisme Canada à Ottawa.

\section{Tourisme et information}

\section{Journalisme de tourisme}

Fédération internationale des journalistes et ecrivains du tourisme (F.I.J.E.T.): Charte de la FJUET in: Le journaliste de tourisme, no 22 (hiver 1982), p. 15.

Fédération internationale des joumalistes et écrivains du tourisme (F.I.J.E.T.). Le rôle du journaliste et de l'écrivain du tou= risme dans la formation du tourisme moderne, Rapport des XVIIle congrès et assemblée générale de la FIJET, (Roumanie, 29 sept. au 9 oct. 1973), 161 p.

MARTIN, Marie, Destination: voyages. Raid éclair sur les chroniques de voyages, in Les diplômés, no 338 (mars-avril 1982), p.p. 23-25.

On trouvera soulevés dans de brefs éditoriaux des questions d'éthique et des problèmes rencontrés dans la pratique du journalisme de tourisme. Voir par exemple:

CAZELAIS, Normand, L'information tou= ristique, une publicité déguisée? in Le Jour, 20 juillet 1974, p. V5.
CAZELAIS, Normand, Linformation touristique concernant la Rhodesie et / Afri. que du Sud in Le Jour, 10 janvier 1976, p. 22.

HAULOT, Arthur, L'information touristi: que: une sincerite sans concession, in Un certain tourisme, Fédération du tourisme de la Province de Hainaut, 1983; p. 99.

HAULOT, Arthur, Oul a /'information, non au bourrage de crâne in: Vue touristique, no 3 (1984), pp. 10-11.

LEROUX, Rémy R., Journalistes de tourisme, crise et développement in: Vue touristique, no 3 (1983), p. 3.

LEROUX, Rémy R. Les joumalistes de tourisme sont ils bons pour le tourisme? in: Vue touristique, no 47 (1981), p. 3.

LEROUX, Rémy R., Plaidoyer pour la critique touristique in: Vue touristique, no 2 (1983), p. 3.

LEROUX, Rémy R., Ouel tourisme demain? La charte d'Acapulco mise sur la presse in: Vue touristique, no 1 (1983), p. 3.
ZOBEL, L.P., Travel Writter Handbook, Manion (Ohio): Writer's digest, 1980, 274 p.*

\section{Information touristique: image projetée et attitude du récepteur} CAZELAIS, Normand, A propos d'une certaine publicite - le touriste n'est-il qu'un crétin? in: Le Jour, 21 aoūt 1976. p. 19.

EHEMANN, Jane, What kind of place is Ireland: an image perceived through the american media in: Journal of travel research, vol. XVI no 2 (fall 1977), p.p.28-30.

GITELSON, Richard J. and John L, Crompton, The planning horizons and sources of information used by pleasure vacationers in: Journal of travel research, vol. XXI no 3 (winter 1983) p.p.2-7.

NOLAN, Sidney D., Tourists "use and evaluation of travel information sources: summary and' conc/usions in: Journal of travel research, vol. XIV, no 3 (winter 1976), p.p.6-8. 
TRONCALLI, Michael T. and John R. Thompson, Relative importance of information sources used by travel opinion Veaders in: Journal of travel research, vol. XIV, no 4 (spring 1976), p.p.11-14.

Traveler information needs, Springfield (UA): US department of transportation, 1977, 77p.*

\section{Information touristique gouvernementale}

Canada, Office de tourisme, Information bulletins for the travel industry branch. Ottawa, oct. 1973-1974*,

LAWSON, J., Canadian travel information study, Ottawa, oct. 1978, 61p."

\section{Les guides}

BARTHES, Roland, Mythologies, Paris, Seuil, 1957, p.p.122 et suivantes.

CAZELAIS, Normand, Des guides touristiques du Quebec: /e meilleur et le pire. in: Le Jour, 3 juillet 1976, p. 19.

CAZELAIS, Normand, Le Klondike des guides de voyage, in: Livre d'ici, mars 1983 , p. 4.
GRIMES, Paul, A guide to how guide books word in: Globe and Mail, 19 jan. 1985 , p.t9.

GRITTI, Jules, Les contenus cu/ture/s du Guide bleu in: Communications (spécial vacances et tourisme) no 10 (1976), Paris, Seuil.

LERIVRAY, Bernard, Guides bleus, guides verts et lunettes roses, Paris, Cerf, $1975,158 \mathrm{p}$.

SLADE, Margot, A guide to guide books in: New-York Times, 1 avril 1984, p.3.

\section{Information touristique et protection du consommateur}

Organisation mondiale du tourisme, Pro= tection et information du touriste en tant que consommateur, rapport présenté lors de l'assemblée générale de Rome, sept. $1981,54 \mathrm{p}$.

\section{Information touristique et nouvelles technologies: vidéo-texte et télématique}

Focus on technology and telecommunications in the travel industry. Tourism management (numéro spécial) vol. 4 , no 4 (Décembre 1983).

Informatique et télématique touristique. Espaces (numéro spécial) no 69 , août 1984.

Organisation mondiale du tourisme, Étude sur I'adaptation de technologies nouvelles du tourisme: la télématique comme cas d'espèces, rapport présenté lors de l'assemblée générale de New-Delhi, oct. $1983,42 p$.

BERG, Eric N., Practical traveler: help from the home computer in: New-York Times, 20 jan. 1985, p.3.

\section{Le voyage comme processus d'information}

HANSEN-STURN, Cord. D., Info-travel: travel as an information medium in: Travel research: the catalyst for worlwide tourism marketing (TTRA 15th annual conference proceedingsl, Salt Lake city, TTRA, p.p.259-266.

\section{Inventaire des médias touristiques}

\section{Les quotidiens}

On trouvera dans ce deuxième volet un aperçu de toute l'information touristique disséminée, au fil de l'actualité, dans la presse écrite:

Centre d'études du tourisme, Revue de presse mensuelle, publiée depuis juillet 1984.

De plus les quotidiens suivants publient une fois la semaine une sélection ou un cahier consacrés aux voyages et au tourisme:

Le Devoir

Le Dimanche-Matin

The Gazette

The Globe and Mail

Le journal de Montréal

Le Monde

The New-York Times

La Presse

Le Soleil

Ces "cahiers" sont conservés et indexés au C.E.T.

\section{Les magazines}

Plusieurs magazines se spécialisent dans I'information et le reportage touristiques ou géographiques:

Atlas

GEO

National geographic traveler

Travel and leasure

Travel Holiday

Vue touristique, etc...
Au Québec la revue Évasion a rempli cette fonction pendant quelques années. On assiste présentement dans ce domaine à une sorte d'éclatement: des reportages et chroniques touristiques se retrouvent dans des magazines de tous genres: de l'Actualité à Clin d'oeil en passant par Chatelaine.

\section{Les périodiques spécialisếs de l'industrie touristique ("Trade"). \\ Bulletin Voyages \\ Canadian travel courrier \\ Canadian travel news \\ Canadian travel press \\ Journal des voyages \\ Marketing voyages \\ Tourisme Plus}

Une liste de l'ensemble des publications de ce type a l'échelle internationale serait trop longue pour être compilée ici. Elle pourrait comprendre également une série de périodiques s'adressant à un segment seulement de l'industrie comme, par exemple, le voyage d'affaires et les congrès (Meetings and conventions, Successfull meetings, etc....).

\section{"Newsletters" spécialisées}

Depuis quelques années on assiste à une prolifération de petites publications genre "Newsletters" qui tentent de répondre aux besoins d'information de voyageurs aux intérêts plus spécifiques. On trouvera une présentation et une liste de ces publications dans l'article suivant:

GRIMES, Paul, Practical Traveler: Newsletter can provide valuable analysis in New-York Times, 27 mai 1984, p.3

On pourra également consulter au C.E.T. la collection complète de La lettre touristique ainsi que des exemplaires des deux "newsletters" suivantes:

Travel advisor

Travel smart. 